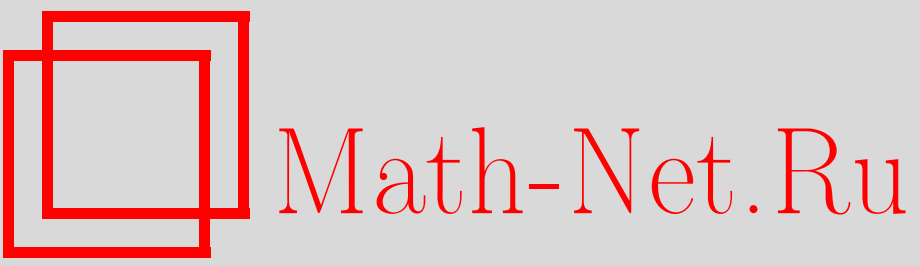

С. Ю. Славянов, Изомонодромные деформации уравнений класса Гойна и уравнения Пенлеве, ТМФ, 2000, том 123, номер 3, 395-406

DOI: https://doi.org/10.4213/tmf611

Использование Общероссийского математического портала Math-Net.Ru подразумевает, что вы прочитали и согласны с пользовательским соглашением

http://www.mathnet.ru/rus/agreement

Параметры загрузки:

IP: 54.162 .127 .20

26 апреля 2023 г., 10:14:38 
ТЕОРЕТИЧЕСКАЯ

И МАТЕМАТИЧЕСКАЯ

ФИЗИКА

Том 123, № 3

июнь, 2000

(C) 2000 г.

С. Ю. Славянов*

\section{ИЗОМОНОДРОМНЫЕ ДЕФОРМАЦИИ УРАВНЕНИЙ КЛАССА ГОЙНА И УРАВНЕНИЯ ПЕНЛЕВЕ}

Статья дополняет две предыдущие публикации автора, посвященные связям между уравнениями классов Гойна и Пенлеве. Завершены формулировка и доказательство теоремы, описывающей эти связи. Дана гамильтонова интерпретация условия изомонодромной деформации. Предложена альтернативная классификация уравнений Пенлеве в составе десяти уравнений.

\section{1. ВВЕДЕНИЕ}

В 1889 г. Гойн написал статью о решениях фуксова обыкновенного дифференциального уравнения (ОДУ) второго порядка с четырьмя регулярно особыми точками [1]. В последующем это уравнение обычно называлось уравнением Гойна. Через 100 лет Зеегер, Ронво и Лай организовали юбилейное рабочее совешание по уравнению Гойна [2], задачей которого был обзор современного состояния в области исследований решений уравнения Гойна. Одним из итогов совешания стало признание того, что, помимо собственно уравнения Гойна, следует изучать его конфлюэнтные формы. Эти конфлюэнтные формы (а именно четыре такие формы) были введены и исследованы ранее в работах $[3,4]$. Более точная классификация уравнений класса Гойна на основе понятия s-ранга особенностей была предложена позднее [5, 6]. Эта классификация включает так называемые редуцированные конфлюэнтные формы уравнения Гойна и различает 10 уравнений, принадлежаших классу Гойна.

В 1898 г. Пенлеве начал серию работ [7], в которых ввел класс нелинейных ОДУ второго порядка, у которых подвижные особенности могут быть только полюсами. Этот класс уравнений сейчас известен как уравнения Пенлеве. Полный список уравнений Пенлеве был получен Гамбье в работе [8] и в несколько отличных обозначениях Гарнье в [9]. Различные решения уравнений Пенлеве образуют класс специальных функций нелинейной математической физики - так называемые трансценденты Пенлеве.

Еще до того, как список уравнений Пенлеве был завершен, Фукс [10] обнаружил замечательную связь между уравнением Гойна и уравнением Пенлеве, известном сейчас

\footnotetext{
* Санкт-Петербургский государственный университет, Санкт-Петербург, Россия. E-mail:slav@slav.usr.pu.ru
} 
как уравнение $P^{V I}$. Он добавил в уравнение Гойна члены, соответствуюшие дополнительной, ложной особенности. Ложной особенностью называется регулярно особая точка линейного ОДУ, в окрестности которой общее решение этого ОДУ является голоморфной функцией. В результате Фукс получил уравнение $P^{V I}$ как условие совместности, состоящее в том, что предложенное уравнение и дополнительное линейное уравнение с дифференцированием по параметру первого уравнения имеют обшие решения. Однако исследования Фукса не получили дальнейшего развития. Позднее становится популярной иная связь между уравнениями Пенлеве и линейными уравнениями (точнее, линейными системами), которую получил Шлезингер [11] и которая в современном виде исследована в работах $[12,13]$. Основанный на этой связи метод сейчас известен как метод изомонодромных деформаций (метод деформаций, сохраняющих монодромию), его изложение приведено, например, в книге [14].

В статье [15] было показано, что уравнения Пенлеве можно рассматривать как ньютоновские уравнения движения, если соответствуюшие квантовые гамильтонианы определены с помощью уравнений Гойна. Этот результат объясняет ранее известную гамильтонову природу уравнений Пенлеве $[14,16,17]$.

Несколько проблем осталось нерешенным в работе [15]. Во-первых, это вопрос о взаимной одназначности соответствия между уравнениями Гойна и Пенлеве. Во-вторых, остался нерешенным также вопрос об альтернативной классификации уравнений Пенлеве с точки зрения теории уравнений Гойна. Эти вопросы получили решение в статье [18]. Однако остался еше один вопрос: какова связь между методом изомонодромных деформаций для уравнений Пенлеве и гамильтоновым подходом к этим уравнениям, предложенным в работе [15]? Обсуждению этого вопроса и посвящена данная публикация. Ответ на него стимулирован работой [10] и основан на деформации уравнений класса Гойна добавлением в уравнение ложной сингулярности.

Напомним сначала основные результаты работы [15]. Каждое уравнение, принадлежащее классу Гойна, может быть представлено в виде

$$
\frac{1}{f(t)}\left[P_{0}(z, t) D^{2}+P_{1}(z, t) D+P_{2}(z, t)\right] y(z)=\lambda y(z), \quad D:=\frac{d}{d z} .
$$

В уравнении (1) $P_{0}(z, t), P_{1}(z, t), P_{2}(z, t)$ - полиномы по $z, t$ - скейлинговый параметр, $\lambda$ - акцессорный параметр. Если квантовым наблюдаемым $\hat{q}, \hat{p}$ (координата и импульс соответственно) поставить в соответствие умножение на $z$ и дифференцирование $D$ в уравнении (1), то последнее приобретает гамильтонову структуру и может быть переписано в виде

$$
H(\hat{q}, \hat{p}, t) y=\lambda y
$$

где

$$
H(\hat{q}, \hat{p}, t)=\frac{1}{f(t)}\left[P_{0}(\hat{q}, t) \hat{p}^{2}+P_{1}(\hat{q}, t) \hat{p}+P_{2}(\hat{q}, t)\right] .
$$

В уравнении (2) функция $H$ является гамильтонианом, адиабатически зависящим от параметра $t$, который можно рассматривать как время ${ }^{1}, \lambda$ - энергия системы. Соответ-

\footnotetext{
1) Физический смысл параметра $t$ может быть различен в конкретных задачах.
} 
ствующий гамильтониан в классической механике квадратичен по классическому импульсу $p$,

$$
H(q, p, t)=\frac{1}{f(t)}\left[P_{0}(q, t) p^{2}+P_{1}(q, t) p+P_{2}(q, t)\right] .
$$

$\mathrm{K}$ этому гамильтониану можно применить преобразование Лежандра, заменяющее импульс $p$ на скорость $q_{t}$. Соответствуюший лагранжиан $\mathcal{L}\left(q, q_{t}, t\right)$, квадратичньй по $q_{t}$, выглядит следуюшим образом:

$$
\mathcal{L}\left(q, q_{t}, t\right)=\frac{f(t)}{4 P_{0}(q, t)}\left(q_{t}-\frac{P_{1}(q, t)}{f(t)}\right)^{2}-\frac{P_{2}(q, t)}{f(t)} .
$$

Выпишем уравнения движения Эйлера-Лагранжа, отвечающие этому лагранжиану,

$$
\begin{aligned}
q_{t t}= & \frac{1}{2} \frac{\partial}{\partial q}\left(\ln P_{0}(q, t)\right) q_{t}^{2}-\left(\frac{\partial}{\partial t}(\ln f(t))-\frac{\partial}{\partial t}\left(\ln P_{0}(q, t)\right)\right) q_{t}+ \\
& +\frac{P_{0}(q, t)}{f^{2}(t)}\left(\frac{\partial}{\partial q} \frac{P_{1}^{2}(q, t)}{2 P_{0}(q, t)}+f(t) \frac{\partial}{\partial t} \frac{P_{1}(q, t)}{P_{0}(q, t)}-2 \frac{\partial P_{2}(q, t)}{\partial q}\right) .
\end{aligned}
$$

Для любого конкретного уравнения, принадлежашего классу Гойна, уравнение (5) является уравнением Пенлеве (доказывается непосредственными вычислениями), обладаюшим свойством Пенлеве. Это означает, что подвижные особенности всех решений уравнения являются полюсами, т.е. нет подвижных точек ветвления или подвижных существенных особых точек.

В действительности в работе [15] было доказано обратное утверждение, что любое уравнение Пенлеве может быть получено как уравнение классического движения, соответствуюшее уравнению класса Гойна. С другой стороны, вычисления, представленные в работах [15] и [18], были достаточны для доказательства прямого и обратного утверждений.

\section{2. ИЗОМОНОДРОМНЫЕ ДЕФОРМАЦИИ}

По причинам, которые станут ясными ниже, необходимо рассматривать само уравнение Гойна и его конфлюэнтные формы отдельно. Для каждого уравнения можно ввести ложную сингулярность, но соответствуюшие члены слегка различаются.

Рассмотрим фуксово дифференциальное уравнение с пятью особенностями, одна из которых $z=q$ является ложной сингулярностью,

$$
\begin{aligned}
D^{2} y(z) & +\left(\frac{c}{z}+\frac{d}{z-1}+\frac{e}{z-t}-\frac{1}{z-q}\right) D y(z)+ \\
& +\left(\frac{a b}{z(z-1)}+\frac{h t(t-1)}{z(z-1)(z-t)}+\frac{p q(q-1)}{z(z-1)(z-q)}\right) y(z)=0 .
\end{aligned}
$$

Будем назьвать это уравнение деформированным уравнением Гойна. Оно отличается от уравнения Гойна двумя дополнительными членами, имеюшими простые полюсы 
при $z=q$. Один из этих членов (с фиксированным вычетом) стоит в коэффициенте перед первой производной, а второй (с произвольным вычетом) - в коэффициенте перед функцией. Наше уравнение соответствует следуюшему символу Римана:

$$
\left(\begin{array}{ccccc}
0 & 1 & t & q & \infty \\
0 & 0 & 0 & 0 & a \\
1-c & 1-d & 1-e & 2 & b
\end{array}\right)
$$

Выполняется фуксово соотношение

$$
c+d+e=a+b+3
$$

Несколько специфическое введение параметров $p$ и $h$ может быть оправдано простотой соотношений

$$
\begin{aligned}
& h=\operatorname{Res}_{z=t} \frac{h t(t-1)}{z(z-1)(z-t)}, \\
& p=\operatorname{Res}_{z=q} \frac{p q(q-1)}{z(z-1)(z-q)},
\end{aligned}
$$

так что $h$ и $p$ - вычеты двух членов в уравнении (6) в особых точках $z=t$ и $z=q$ соответственно. То, что точка $z=q$ является ложной особенностью, справедливо, если уравнение (6) имеет локальное решение, разложение Тейлора которого есть

$$
y(z)=\sum_{k=0}^{\infty} g_{k}(z-q)^{k}, \quad g_{0}=1
$$

с произвольным коэффициентом $g_{2}$. Введем следуюшие обозначения:

$$
\begin{gathered}
P(z)=\frac{c}{z}+\frac{d}{z-1}+\frac{e}{z-t}, \quad Q(z)=\frac{a b}{z(z-1)} \\
f(t)=t(t-1), \quad \rho(z)=z(z-1)(z-t)
\end{gathered}
$$

Тогда уравнение (6) можно записать как

$$
D^{2} y(z)+\left(P(z)-\frac{1}{z-q}\right) D y(z)+\left(Q(z)+\frac{h f(t)}{\rho(z)}+\frac{p q(q-1)}{z(z-1)(z-q)}\right) y(z)=0
$$

Подставляя разложение (8) в уравнение (9), приходим к рекуррентным уравнениям для коэффициентов $g_{k}$ этого разложения. В частности, для $g_{1}$ и $g_{2}$ имеем

$$
\begin{gathered}
p_{-1} g_{1}+q_{-1}=0 \\
2\left(1-p_{-1}\right) g_{2}+\left(p_{0}+q_{-1}\right) g_{1}+q_{0}=0
\end{gathered}
$$


здесь $p_{k}, q_{k}, k=-1,0,-$ коэффициенты разложения Лорана функций $P(z), Q(z)$ в точке $z=q$ соответственно,

$$
P(z)=\sum_{k=-1}^{\infty} p_{k}(z-q)^{k}, \quad Q(z)=\sum_{k=-1}^{\infty} q_{k}(z-q)^{k} .
$$

Их значения вычисляются непосредственно из уравнения (6),

$$
\begin{gathered}
p_{-1}=-1, \quad q_{-1}=p \\
p_{0}=\frac{c}{q}+\frac{d}{q-1}+\frac{e}{q-t} \\
q_{0}=\frac{a b}{q(q-1)}-p\left(\frac{1}{q}+\frac{1}{q-1}\right)+\frac{h t(t-1)}{q(q-1)(q-t)} .
\end{gathered}
$$

Учитывая введенные выше обозначения, уравнения (11) запишем в виде

$$
\begin{gathered}
p_{-1}=-1, \quad q_{-1}=p \\
p_{0}=P(q), \\
q_{0}=Q(q)-p\left(\frac{1}{q}+\frac{1}{q-1}\right)+\frac{h f(t)}{\rho(q)},
\end{gathered}
$$

уравнения (10) принимают тогда вид

$$
\begin{gathered}
g_{1}=p \\
0 \cdot g_{2}+(P(q)+p) p+Q(q)-p\left(\frac{1}{q}+\frac{1}{q-1}\right)+\frac{h f(t)}{\rho(q)}=0
\end{gathered}
$$

Так как множитель перед $g_{2}$ равен нулю, мы приходим к условию ${ }^{2)}$ того, что точка $z=q$ должна являться ложной сингулярностью. Это условие приводит к следуюшему соотношению для параметров $h, p, q, t$ :

$$
h(q, p, t)=-\frac{\rho(q)}{f(t)}\left[p^{2}+\left(P(q)-\frac{1}{q}-\frac{1}{q-1}\right) p+Q(q)\right]
$$

или

$$
\begin{aligned}
h(q, p, t)= & \frac{1}{t(t-1)}\left[q(q-1)(q-t) p^{2}+((c-1)(q-1)(q-t)+\right. \\
& +(d-1) q(q-t)+e q(q-1)) p+a b(q-t)] .
\end{aligned}
$$

Очевидно, что функция $h(q, p, t)$ совпадает с гамильтонианом, соответствующим уравнению Гойна с точностью до сдвига в параметрах $c$ и $d$ на единицу, т.е.

$$
h(q, p, t, a, b, c, d, e)=H^{(1,1,1 ; 1)}(q, p, t, a, b, c-1, d-1, e) .
$$

\footnotetext{
2) Здесь рассматривается частный случай, когда разность характеристических экспонент в точке $q$ равна двум.
} 
Это объясняется тем, что параметры $a, b, c, d, e$ удовлетворяют различным фуксовым условиям в случае уравнения Гойна и в случае деформированного уравнения Гойна.

Предположим, что $h(p, q, t)$ - гамильтониан для некоторой одномерной классической динамической системы. Импульс частицы обозначим $p$, координату $-q$ и время $-t^{3)}$. Классическое движение частицы определяется гамильтоновыми уравнениями

$$
\frac{d p}{d t}=-\frac{\partial h}{\partial q}, \quad \frac{d q}{d t}=\frac{\partial h}{\partial p}
$$

С другой стороны, уравнения (13)-(15) могут быть рассмотрены как условия деформаиий, сохраняющих монодромию ${ }^{4)}$. Очевидно, что вычет в точке $z=t$, так же как и вычеты в точках $z=0, z=1$, не зависит от поведения функций $p(t), q(t)$ при выполнении уравнений (15).

Уравнения (15) эквивалентны (после соответствующего преобразования Лежандра) нютоновскому уравнению движения, которое является уравнением Пенлеве $P^{V I}$, как это было отмечено в предыдушем разделе. Сдвиг в параметрах и несколько специфическое выражение для деформирующего члена (7) объясняются фуксовой структурой уравнения Гойна.

В обшей ситуации произвольного уравнения, принадлежашего классу Гойна, за исключением самого уравнения Гойна, мы записываем это уравнение в форме

$$
D^{2} y(z)+P(z) D y(z)+\left(Q(z)+\frac{f(t) h}{\rho(z)}\right) y(z)=0
$$

Деформированное уравнение с дополнительной ложной сингулярностью в точке $z=q$ имеет вид

$$
D^{2} y(z)+\left(P(z)-\frac{1}{z-q}\right) D y(z)+\left(Q(z)+\frac{f(t) h}{\rho(z)}+\frac{p}{z-q}\right) y(z)=0
$$

Функция $f(t)$ определяется в процессе конфлюэнции. Ее выбор для различных уравнений был показан в предыдущем разделе.

Условие, что уравнение (17) имеет локальное решение в форме (8), приводит к уравнениям для первых двух коэффициентов

$$
\begin{gathered}
-g_{1}+p=0 \\
(P(q)+p) p+\left(Q(q)+\frac{f(t) h}{\rho(q)}\right)=0
\end{gathered}
$$

\footnotetext{
${ }^{3)}$ Во многих практических приложениях параметр $t$ имеет другой физический смысл. К примеру, в двухцентровой кулоновской задаче $t$ имеет смысл расстояния между центрами.

${ }^{4)}$ Они также называются изомонодромными условиями.
} 
Первое из этих уравнений можно интерпретировать как оператор дифференцирования, действуюший подобно умножению на классический импульс $p$. Второе из этих уравнений можно интерпретировать как подстановку независимой переменной $z$ вместо классической координаты $q$ в уравнение класса Гойна. В качестве условия совместности получается следуюшее соотношение для $h, p, q, t$ :

$$
h(p, q, t)=-\frac{1}{t} \rho(q)\left(p^{2}+P(q) p+Q(q)\right),
$$

т.е. снова получается классический гамильтониан, соответствующий уравнению класса Гойна. Условия деформаций, сохраняющих монодромию, отвечают соответствующему уравнению класса Гойна и имеют вид гамильтоновой системы (15). В каждом случае эта система эквивалентна соответствуюшему уравнению Ньютона, которое имеет второй порядок и оказывается одним из уравнений Пенлеве. Эти уравнения будут выписаны в разделе 3 .

В результате мы приходим к следуюшей теореме.

ТеоремА. Каждое деформированное уравнение класса Гойна с добавленной ложной сингулярностью связано через условие деформаций, сохраняющих монодромию, с уравнением Пенлеве. Каждое уравнение Пенлеве можно рассматривать как условие деформаций, сохраняющих монодромию, для деформированного уравнения класса Гойна.

Альтернативная возможность получить уравнения Пенлеве как изомонодромные условия состоит в использовании систем уравнений первого порядка вместо линейных ОДУ второго порядка. Такой подход был предложен в работе [11]. Мы приведем здесь набросок подхода к уравнениям Пенлеве на основе систем Шлезингера.

Для уравнения $P^{V I}$ соответствуюшая система Шлезингера имеет вид

$$
\vec{\Psi}_{z}=A(z, t) \vec{\Psi}
$$

где $\vec{\Psi}$ - двумерный вектор, $A(z, t)$ - матрища вида

$$
A(z, t)=\frac{A^{0}}{z}+\frac{A^{1}}{z-1}+\frac{A^{t}}{z-t},
$$

отражающего структуру сингулярностей уравнения (19). Здесь $A^{0}, A^{1}, A^{t}$ - матрищы с постоянными коэффициентами. Собственные числа этих матрищ являются характеристическими показателями соответствующих особенностей. Для явных выгислений, однако, необходимо параметризовать матричные элементы матриц $A^{0}, A^{1}, A^{t}$ в терминах параметров $a, b, c, d, e, t$. Предполагается также, что в бесконечно далекой точке матрица $A(z, t)$ упрошается и становится диагональной матрицей $A^{\infty}$, т.е.

$$
\begin{gathered}
\lim _{z \rightarrow \infty} A(z, t)=-\frac{A^{\infty}}{z} \\
A^{\infty}=-\left(A^{0}+A^{1}+A^{t}\right), \quad A^{\infty}=\left(\begin{array}{cc}
\kappa_{1} & 0 \\
0 & \kappa_{2}
\end{array}\right) .
\end{gathered}
$$


Вместе с системой (19) изучается и другая система

$$
\vec{\Psi}_{t}=B \vec{\Psi}, \quad B:=\frac{A^{t}}{t-z} \vec{\Psi} .
$$

Необходимо ввести определенные условия для $\vec{\Psi}$. А именно при $z \rightarrow \infty$

$$
\vec{\Psi}(z, t) \rightarrow\left(\begin{array}{cc}
z^{\kappa_{1}} & 0 \\
0 & z^{\kappa_{2}}
\end{array}\right)
$$

Тогда условие изомонодромных деформаций имеет вид

$$
A_{t}-B_{z}+[A, B]=0 .
$$

Это условие совпадает с уравнением $P^{V I}$. Хотя схема рассуждений проста, реальная параметризация матричных элементов весьма громоздка и требует нескольких страниц только для представления результата. Объяснение нормировки на бесконечности также нетривиально.

Ложная сингулярность для линейного ОДУ второго порядка “спрятана" в системе (19). Она соответствует нулю недиагонального матричного элемента матришы $A(z, t)$. Матрицы $A, B$ называются парой Лакса для соответствующего уравнения Пенлеве. Аналогичным образом можно рассмотреть все другие уравнения Пенлеве.

Учитывая оба подхода, представленных здесь и в статье [15], мы можем сформулировать предложение, включающее обе интерпретации уравнений Пенлеве в терминах уравнений Гойна.

ПРЕДЛОЖЕНИЕ. Каждъй тип уравнений класса Гойна соответствует уравнению класса Пенлеве так же, как уравнение Шредингера соответствует уравнению движения в классической динамике. Обратное утверждение также справедливо: каждый тип уравнения Пенлеве может быть получен с помощью соответствующего уравнения Гойна.

\section{3. КЛАССИФИКАЦИЯ}

Принципы классификации уравнений Гойна на основе процессов конфлюэнции были предложены в работе [5] и полностью описаны в работе [18]. Такая классификация различает:

1. Основное уравнение Гойна - УГ.

2. Четыре типа конфлюэнтных случаев:

- конфлюэнтное уравнение Гойна (КУГ), уравнение с двумя регулярно особыми точками и одной иррегулярно особой точкой на бесконечности;

- биконфлюэнтное уравнение Гойна (БКУГ), уравнение с одной регулярно особой точкой и одной иррегулярно особой точкой на бесконечности, возникающей в результате слияния трех регулярно особых точек;

- дважды конфлюэнтное уравнение Гойна (ДКУГ), уравнение с двумя регулярно особыми точками, одна в нуле и вторая на бесконечности; 
- триконфлюэнтное уравнение Гойна (ТКУГ), одна иррегулярно особая точка на бесконечности, возникаюшая в результате слияния четырех регулярно особых точек.

3. Пять типов редуцированных конфлюэнтных случаев:

- редуцированное конфлюэнтное уравнение Гойна (РКУГ);

- редуцированное биконфлюэнтное уравнение Гойна (РБКУГ);

- редуцированное дважды конфлюэнтное уравнение Гойна (Р ДКУГ);

- редуцированное триконфйюэнтное уравнение Гойна (РТКУГ);

- дважды редуцированное дважды конфлюэнтное уравнение Гойна (ДР ДКУГ).

В редуцированных уравнениях решения в окрестности иррегулярной особой точки раскладываются не по целым, а по полуцелым степеням аргумента.

Ниже для удобства ссылок мы приведем уравнения класса Гойна и соответствующие им уравнения, принадлежащие классу Пенлеве. В некоторых, но не во всех случаях они написаны в обозначениях, применяемых обычно в теории уравнений Пенлеве. Каждое уравнение класса Гойна представлено гамильтонианом, после которого выписано соответствуюшее уравнение Пенлеве:

УГ:

$$
\begin{aligned}
H(q, D, t)= & \frac{-1}{t(t-1)}\left[q(q-1)(q-t) D^{2}+(c(q-1)(q-t)+\right. \\
& +d q(q-t)+(a+b+1-c-d) q(q-1)) D+a b q] \\
q_{t t}= & \frac{1}{2}\left(\frac{1}{q}+\frac{1}{q-1}-\frac{1}{q-t}\right) q_{t}^{2}-\left(\frac{1}{t}+\frac{1}{t-1}+\frac{1}{q-t}\right) q_{t}+ \\
& +\frac{q(q-1)(q-t)}{t^{2}(t-1)^{2}}\left(\frac{(a+b-1)^{2}}{2}-2(a-1)(b-1)-\frac{c^{2} t}{2 q^{2}}+\right. \\
& \left.+\frac{d^{2}(t-1)}{2(q-1)^{2}}+\frac{\left(1-(2+c+d-a-b)^{2}\right) t(t-1)}{(q-t)^{2}}\right) .
\end{aligned}
$$

С точностью до обозначений коэффициентов уравнение (23) совпадает с уравнением $P^{V I}$.

КУГ:

$$
\begin{aligned}
H(q, D, t)= & \frac{-1}{t}\left[q(q-1) D^{2}+(-t q(q-1)+c(q-1)+d q) D-a t q\right], \\
q_{t t}= & \frac{1}{2}\left(\frac{1}{q}+\frac{1}{q-1}\right) q_{t}^{2}-\frac{1}{t} q_{t}+\frac{1}{t^{2}}\left(\frac{c^{2}(q-1)}{2 q}-\frac{d^{2} q}{2(q-1)}\right)+ \\
& +\frac{q(q-1)(2 q-1)}{2}-\frac{q(q-1)}{t}(1+c+d-2 a) .
\end{aligned}
$$

Уравнение (25) может быть преобразовано к специальному случаю уравнения $P^{V}$.

БКУГ:

$$
\begin{aligned}
& H(q, D, t)=-\left(q D^{2}+(-q(q+t)+c) D-a q\right) \\
& q_{t t}=\frac{q_{t}^{2}}{2 q}+\frac{3}{2} q^{3}+2 t q^{2}+\left(\frac{t^{2}}{2}+2 a-c\right) q-\frac{c^{2}}{2 q}
\end{aligned}
$$


С точностью до обозначений коэффициентов и растяжения переменных уравнение (27) совпадает с уравнением $P^{I V}$.

ДКУГ:

$$
\begin{gathered}
H(q, D, t)=\frac{-1}{t}\left(q^{2} D^{2}+\left(-q^{2}-t+c q\right) D-a q\right), \\
q_{t t}=\frac{q_{t}^{2}}{q}-\frac{q_{t}}{t}+\frac{1}{q}+\frac{q^{3}}{t^{2}}+\frac{q^{2}(2 a-c)}{t^{2}}-\frac{c-1}{t} .
\end{gathered}
$$

Уравнение (29) может быть преобразовано к специальному случаю уравнения $P^{I I I}$.

ТКУГ:

$$
\begin{gathered}
H(q, D, t)=-\left(D^{2}+\left(-q^{2}-t\right) D-a q\right), \\
q_{t t}=2 q^{3}+2 t q+2 a .
\end{gathered}
$$

С точностью до обозначений коэффишиентов и растяжения параметров уравнение (31) совпадает с уравнением $P^{I I}$.

РКУГ:

$$
\begin{aligned}
H(q, D, t)=\frac{-1}{t} & {\left[q(q-1) D^{2}+(c(q-1)+d q) D-t q\right], } \\
P^{(1,1 ; 3 / 2): \quad q_{t t}=} & \frac{1}{2}\left(\frac{1}{q}+\frac{1}{q-1}\right) q_{t}^{2}-\frac{1}{t} q_{t}+ \\
& +\frac{1}{t^{2}}\left(\frac{c^{2}(q-1)}{2 q}-\frac{d^{2} q}{2(q-1)}\right)+\frac{2 q(q-1)}{t} .
\end{aligned}
$$

Уравнение (33) может быть преобразовано к специальному случаю уравнения $P^{V}$. Взятые вместе, уравнения $(25),(33)$ покрывают все модифиикации уравнения $P^{V}$.

РБКУГ:

$$
\begin{gathered}
H(q, D, t)=-\left(q D^{2}+c D-t q\right), \\
q_{t t}=\frac{q_{t}^{2}}{2 q}-\frac{c^{2}}{2 q}+4 q^{2}-2 t q .
\end{gathered}
$$

Уравнение (35) может быть преобразовано к уравнению $P^{I I}$.

Р ДКУГ:

$$
\begin{gathered}
H(q, D, t)=\frac{-1}{t}\left(q^{2} D^{2}-q^{2} D-a q-\frac{t}{q}\right), \\
q_{t t}=\frac{q_{t}^{2}}{q}-\frac{q_{t}}{t}+\frac{2 a q^{2}+q^{3}}{t^{2}}-\frac{2}{t} .
\end{gathered}
$$

ДР ДКУГ:

$$
\begin{gathered}
H(q, D, t)=\frac{-1}{t}\left(q^{2} D^{2}-q-\frac{t}{q}\right), \\
P^{(3 / 2 ; 3 / 2)}: \quad q_{t t}=\frac{q_{t}^{2}}{q}-\frac{q_{t}}{t}+\frac{2 q^{2}}{t^{2}}-\frac{2}{t} .
\end{gathered}
$$


Взятые вместе, уравнения (29), (37), (39) покрывают все модификации уравнения $P^{I I I}$. РТКУГ:

$$
\begin{gathered}
H(q, D, t)=-\left(D^{2}+\left(-q^{3}-t q\right) D\right) \\
P^{(; 7 / 2)}: \quad q_{t t}=6 q^{2}+t .
\end{gathered}
$$

С точностью до обозначений коэффициентов и растяжения переменных уравнение (41) совпадает с уравнением $P^{I}$.

\section{4. ОБСУЖДЕНИЕ РЕЗУЛЬТАТОВ}

Мы полагаем, что представленные соотношения между уравнениями класса Гойна и уравнениями Пенлеве, основанные на физических представлениях, в дальнейшем могут быть использованы в различных приложениях. Так, в терминах решений уравнений Гойна описываются эффект Штарка в водороде, задача $\mathrm{H}_{2}^{+}$в молекулярной физике, модели черных дыр в астрофизике и т.д. Для всех этих задач дополнительная возможность исследования классического аналога позволяет улучшить понимание явления. Мы также полагаем, что представленная здесь альтернативная классификация уравнений Пенлеве упростит представление асимптотического поведения трансцендентов Пенлеве на основе структуры линий Стокса соответствующего уравнения Гойна. $\mathrm{K}$ тому же традиционное изучение асимптотического поведения трансцендентов Пенлеве [19] может быть в рамках нашего подхода технически более простым. Для уравнений класса Гойна есть ряд преобразований и симметрий. Они должны иметь аналоги и для уравнений Пенлеве.

Благодарности. Автор благодарен проф. Л. Бордаг, которая указала, что уравнение (16) принадлежит классу Пенлеве, докт. А. Капаеву, отметившему, что это уравнение может быть преобразовано ко второму уравнению Пенлеве, и докт. В. Лаю за дружескую помощь. Автор также благодарен проф. А. Зеегеру, проф. П. Лески, проф. А. Воросу, проф. Р. Конту и проф. А. Бобенко за поддержку и полезные дискуссии.

Работа была поддержана РФ ФИ, гранты № 96-00-01-618,o. 98-01-01-075, стипендией DFG и поддержкой SFB-288 и СEA.

\section{Список литературы}

[1] K. Heun. Math. Annal. 1889. V. 33. P. 161.

[2] Centennial Workshop on Heun's Equation. Theory and Applications. Eds. A. Seeger, W. Lay. Stuttgart: MPI/MF, 1990.

[3] A. Decarreau, M. C. Dumont-Lepage, P. Maroni, A. Robert, A. Ronveaux. Ann. Soc. Sc. Bruxelles. 1978. V. 92. P. 53.

[4] A. Decarreau, P. Maroni, A. Robert. Ann. Soc. Sc. Bruxelles. 1978. V. 92. P. 151

[5] S. Yu. Slavyanov, W. Lay. A. Seeger. Classification Heun's Differential Equations. Ed. A. Ronveaux. Oxford: Oxford University Press, 1995.

[6] А. Зеегер, В. Лай, С. Ю. Славянов. ТМФ. 1995. Т. 104. С. 233.

[7] P. Painlevé. Bull. C. R. Acad. Sci. Paris. 1898. V. 126. P. 1697; Bull. Soc. Math. France. 1900. V. 28. P. 201; Acta Math. 1902. V. 25. P. 1. 
[8] B. Gambier. Acta Math. 1909. V. 33. P. 1.

[9] R. Garnier. Ann. Sci. Ecole Norm. Sup. 1912. V. 29. P. 1.

[10] R. Fuchs. Math. Ann. 1907. V. 63. P. 301.

[11] L. Schlesinger. J. Reine Angew. Math. 1912. V. 141. P. 96.

[12] M. Jimbo, T. Miwa, K. Ueno. Physica D. 1981. V. 2. P. 306; M. Jimbo, T. Miwa. Physica D. 1981. V. 2. P. 407; V. 4. P. 26.

[13] H. Flashka, A. C. Newell. Commun. Math. Phys. 1980. V. 76. P. 65.

[14] K. Iwasaki, H. Kimura, S. Shimomura, M. Ioshida. From Gauss to Painlevé: A Modern Theory of Special Functions. Braunschweig: Vieweg, 1991.

[15] S. Yu. Slavyanov. J. Phys. A. 1996. V. 29. P. 7329.

[16] J. Malmquist. Ark. Mat. Astr. Fys. 1922-1923. V. 17. P. 1.

[17] K. Okamoto. Ann. Math. Pure Appl. 1986. V. 146. P. 337.

[18] С. Ю. Славянов. ТМФ. 1999. Т. 119. С. 3.

[19] A.R. Its, V. Yu. Novokshenov. The Isomonodromic Deformation Method in the Theory of Painlevé Equations. Lecture Notes in Mathematics. V. 1191. Berlin-Heidelberg, New York-Tokyo: Springer-Verlag, 1986. 